\title{
Uso de retalho de gordura de Bichat no fechamento de defeito ósseo pós-enucleação cística: relato de caso
}

Use of Bichat fat retail in closing bone defect after cystic enucleation: case report

Uso de injerto la bola adiposa de Bichat para cierre del defecto del hueso después de la enucleación cística: reporte de caso Heitor Ferreira de SOUZA NETO ${ }^{1}$ Henrique Lima Ferreira de SOUZA ${ }^{1}$ Lara Marques Magalhães MORENO ${ }^{2}$

Renata de Albuquerque Cavalcanti ALMEIDA ${ }^{3}$

Emerson Filipe de Carvalho NOGUEIRA ${ }^{4}$

${ }^{1}$ Curso de Graduação em Odontologia no Centro Universitário Brasileiro (UNIBRA), 50050-230 Recife - PE, Brasil

${ }^{2}$ Especialista em Prótese Dentária, Mestre em Odontologia. Professora de Clínica Integrada, Centro Universitário Brasileiro (UNIBRA), 50050-230 Recife - PE, Brasil

${ }^{3}$ Especialista, Mestre e Doutora em Cirurgia e Traumatologia Bucomaxilofacial, Professora de Cirurgia Oral, Centro Universitário Brasileiro (UNIBRA), 50050-230 Recife - PE, Brasil

${ }^{4}$ Especialista e Mestre em Cirurgia e Traumatologia Bucomaxilofacial. Professor de Cirurgia Oral, Centro Universitário Brasileiro (UNIBRA), 50050-230 Recife - PE, Brasil

\section{Resumo}

Introdução: O coxim adiposo de Bichat é uma massa adiposa lobulada, de fácil acesso, que pode ser utilizada para correção de defeitos ósseos e complicações cirúrgicas por apresentar: menor morbidade ao sítio doador, rica vascularização sanguínea, confiabilidade, altas taxas de sucesso e não deixa cicatrizes visíveis. Objetivo: Relatar um caso clínico de um paciente que apresentou comunicação bucossinusal após exodontia e remoção de cisto periapical em maxila, em que foi utilizada a gordura de Bichat para correção do defeito ósseo. Relato do caso: Paciente do sexo masculino, 52 anos de idade, apresentou queixa de dor em maxila esquerda e sensação de peso em face. Ao exame tomográfico foi observada imagem sugestiva de cárie profunda no 26 e cisto periapical na região. O tratamento consistiu da exodontia, enucleação cística e fechamento do defeito com a gordura de Bichat, sob anestesia geral. Paciente foi acompanhado por 60 dias sem recidiva da lesão. Conclusão: Por apresentar uma rica vascularização, menor morbidade para o sítio doador e baixas taxas de complicações, o corpo adiposo bucal foi utilizado para o tratamento da comunicação bucossinusal e correção do defeito ósseo, assegurando o sucesso do procedimento.

Descritores: Corpo Adiposo; Fístula Bucal; Sinusite Maxilar.

\section{Abstract}

Introduction: The Bichat fat pad is a lobulated fat mass, easily accessible, that can be used to correct bone defects and surgical complications, for presenting: less morbidity at the donor site, rich blood vascularity, reliability, high success rates and for not leaving visible scars. Objective: Report a clinical case of a patient who presented oroantral comunications after extraction and removal of periapical cyst in the maxilla, in which Bichat's fat was used to correct the bone defect. Case report : A 52-year-old male patient complained pain in the left maxilla and feeling of heaviness in face. The tomographic examination showed an image suggestive of deep caries in the 26 and a periapical cyst in the region. The treatment consisted of extraction, cystic enucleation and closure of the defect by using Bichat's fat, under general anesthesia. The patient was accompanied for 60 days without recurrence of the lesion. Conclusion: Due to its rich vascularization, lower morbidity to the donor site and low rates of complications, the buccal adipose body was used to treat oroantral communication and correct bone defects, ensuring the success of the procedure.

Descriptors: Fat Body; Oral Fistula; Maxillary Sinusites.

\section{Resumen}

La almohadilla de grasa Bichat es una masa de grasa lobulada, de fácil acceso, que puede usarse para corregir defectos óseos y complicaciones quirúrgicas porque presenta: menos morbilidad en el sitio donante, rica vascularización sanguínea, confiabilidad, altas tasas de éxito y no deja cicatrices visibles. Objetivo: informar un caso clínico de un paciente que presentó comunicación oroantral después de la extracción y extracción del quiste periapical en el maxilar, en el que se utilizó la grasa de Bichat para la correción del defecto óseo. Caso clínico: un paciente masculino de 52 años se quejaba de dolor en el maxilar izquierdo y sensación de pesadez en la cara. El examen tomográfico mostró una imagen sugestiva de caries profunda en el 26 y un quiste periapical en la región. El tratamiento consistió en extracción, enucleación quística y cierre del defecto con grasa de Bichat, bajo anestesia general. El paciente fue seguido durante 60 días sin recurrencia de la lesión. Conclusión: debido a su rica vascularización, menor morbilidad en el sitio donante y bajas tasas de complicaciones, el cuerpo adiposo bucal se utilizó para tratar la comunicación oroantral y la corrección del defecto óseo, asegurando el éxito del procedimiento.

Descriptores: Cuerpo Adiposo; Fistula Oral; Sinusitis Maxilar.

INTRODUÇÃO

As comunicações bucossinusais são frequentemente associadas a traumas, patologias e exodontias ${ }^{1-4}$. Quando relacionadas a extrações dentárias, é mais comum na extração de molares superiores, devido à proximidade que as raízes apresentam com o seio maxilar ${ }^{1,2,4}$.

Em casos onde o seio maxilar demostre sinais de infecção, inflamação e/ou a comunicação exceda $3 \mathrm{~mm}$, se faz necessária a intervenção cirúrgica para o fechamento da comunicação ${ }^{2,4,5}$. São variadas as técnicas já utilizadas para a correção da comunicação bucossinusal, no entanto, a escolha de qual tratamento utilizar, varia de acordo com o tamanho e localização da comunicação, quantidade e disponibilidade tecidual para o reparo, presença de processo infeccioso e tempo decorrido até o diagnóstico ${ }^{4,5}$.

O fechamento da comunicação bucossinusal através do reposicionamento da gordura de Bichat, é uma técnica cirúrgica simples, apresenta altas taxas de sucesso e baixas taxas de complicações na literatura, devido ao seu rico suprimento sanguíneo, baixa morbidade do sítio doador, confiabilidade e 
facilidade de coleta $^{4,6}$. Sua técnica cirúrgica consiste em, após o acesso, realiza-se uma incisão no periósteo, na região posterior ao pilar zigomático, em seguida, uma cautelosa dissecção, medial ao processo coronóide, expondo o tecido adiposo com o auxílio de pinças delicadas, até liberação do tecido e avanço do retalho ${ }^{6}$.

O objetivo desse trabalho é relatar um caso de comunicação bucossinusal causado após remoção dentária e enucleação cística de maxila, tratado pela técnica de avanço de retalho de gordura de Bichat.

\section{CASO CLÍNICO}

Paciente do sexo masculino, 52 anos de idade, compareceu ao ambulatório queixandose de dor em face, cefaleia constante e halitose. O mesmo já tinha sido avaliado por um otorrinolaringologista que suspeitou de sinusite odontogênica, prescrevendo assim, amoxicilina $500 \mathrm{mg}$ com clavulanato de potássio $125 \mathrm{mg}$, além do uso de corticoide tópico intranasal, e encaminhando para ambulatório de Cirurgia Bucomaxilofacial. Ao exame físico, pode-se observar elemento dentário 26 com restauração extensa e 27 com discreta mobilidade, com dor a palpação em parede anterior de seio maxilar na mesma região, além da presença de terceiro molar cariado e em infra-oclusão (Figura 1A). A tomografia computadorizada de feixe cônico demonstrou velamento completo de seio maxilar esquerdo, cárie secundária no dente 26 , dente 27 com cárie extensa e lesão de furca, associada a lesão hipodensa em região periapical (Figura 1B). Nova tomografia computadorizada médica foi realizada $e$ observou-se a melhora do velamento, e uma melhor distinção da lesão periapical dentro do seio maxilar esquerdo (Figura 1C). Após avaliação clínica, a hipótese diagnóstica foi de sinusite odontogênica associada a lesão reacional perapical.

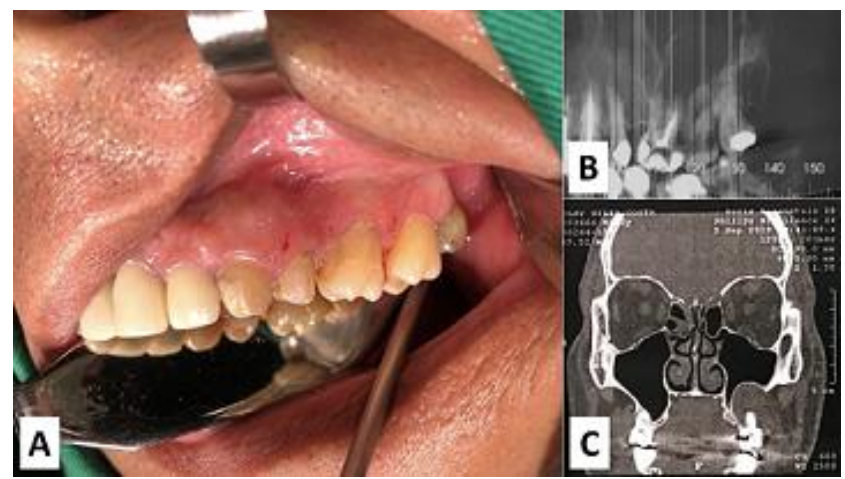

Figura 1: A) Aspecto clínico intraoral. B) Radiografia panorâmica dos maxilares demonstrando imagem sugestiva de cárie extensa do 27, com lesão de furca e cisto em região periapical. C) Tomografia computadorizada após tratamento inicial da sinusite aguda, evidenciando a lesão no seio maxilar esquerdo.
O paciente foi submetido à anestesia geral, onde realizou-se incisão triangular com relaxante anterior (Figura 2A), e após descolamento, foi removido os elementos 27 e 28. Em seguida, foi feita a enucleação cística, a qual causou a comunicação bucossinusal devido ao fino remanescente ósseo que separava a cavidade da lesão e o seio maxilar (Figura 2B). Foi feita osteoplastia na região retirando quaisquer espículas ósseas, e irrigado copiosamente com soro fisiológico $0,9 \%$.

Observado o tamanho do defeito, optouse pela incisão na face interna do retalho, no periósteo, divulsionamento em direção póstero superior até localização da gordura de Bichat. Feito a apreensão com pinça hemostática (Figura 2C), continuou o divulsionamento do tecido até avanço do retalho de gordura e fechamento passivo do defeito ósseo (Figura 2D).

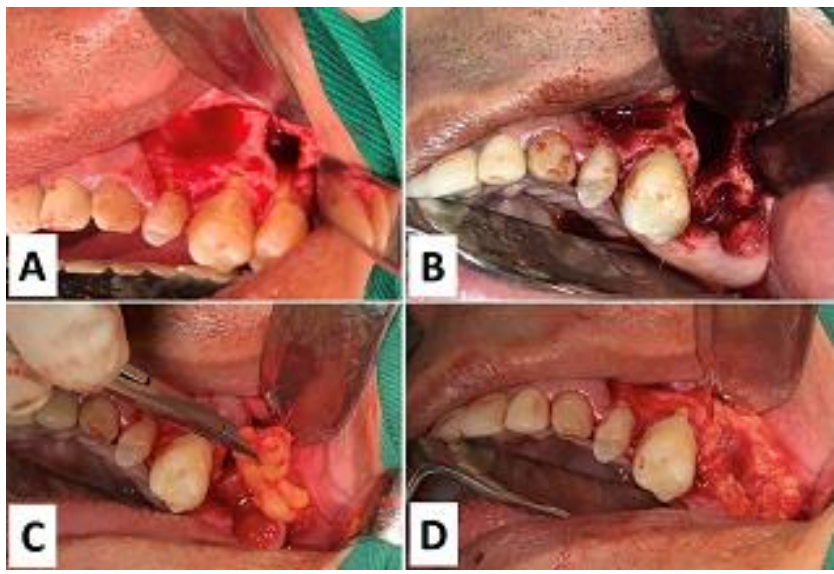

Figura 2: A) Aspecto transoperatório após acesso cirúrgico apresentando exposição radicular do 27 e fenestração óssea. B) Imagem após remoção do dente e enucleação do cisto, com presença da comunicação bucossinusal no ápice do alvéolo. C) Localização, divulsão e avanço do retalho de gordura de Bichat. D) Oclusão e estabilização da gordura sobre o defeito ósseo.

Para manutenção do retalho de gordura em posição, realizou-se uma perfuração no osso e sutura do tecido na perfuração. Todo o retalho cirúrgico foi suturado com monocryl 4-0 (J\&J Medical Devices) (Figura 3A). O paciente foi acompanhado por 60 dias, com cicatrização em normalidade, sem dor, cefaleia ou saída de líquido (Figura 3B).

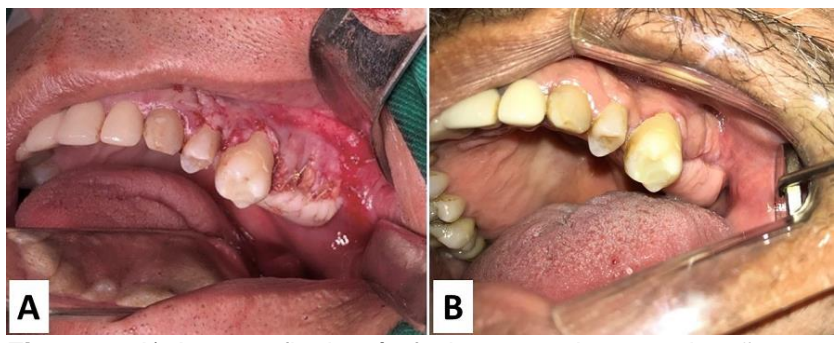

Figura 3: A) Aspecto final após fechamento da comunicação com gordura e sobreposição do retalho bucal. B) Paciente com 2 meses de pós-operatório. 
DISCUSSÃO

A gordura de Bichat foi inicialmente descrita por Heister ${ }^{7}$ em 1732, e Bichat $^{8}$, em 1801, analisou suas características histológicas. O corpo adiposo bucal consiste em uma massa adiposa lobulada, apresentando um feixe central com quatro ramificações, sendo revestida por uma fina capsula fibrosa. Situa-se no espaço mastigatório, entre a região posterior da maxila e as fibras musculares do bucinador, preenchendo áreas adjacentes às musculaturas ${ }^{4,6}$.

O corpo adiposo de Bichat atua como coxim para os músculos mastigatórios, facilitando a movimentação da mastigação, permite a neutralização da pressão negativa da sucção no recém-nascido, abriga e protege estruturas nervosas, além de abrigar ampla rede de vascularização venosa através do plexo pterigóideo ${ }^{3,6,9}$. Geralmente apresenta volume de $10 \mathrm{~cm}^{3}$, sendo um tecido altamente flexível e remodelável ${ }^{6}$. Seu suprimento sanguíneo se dá através dos ramos bucais e temporais profundos da artéria maxilar, além do ramo transverso da face da artéria temporal superficial e pequenos ramos da artéria facial $^{3,4,6,10}$.

O primeiro relato da utilização da gordura de Bichat como enxerto pedicular foi em 1977, por Egyedi ${ }^{9}$, em que utilizou a gordura bucal para correção de comunicação bucossinusal. Vuillemin et al. ${ }^{10}$, Tideman et al. ${ }^{11}$ e outros autores, contribuíram para o estudo da histologia, função, vascularização e volume, ampliando o conhecimento do corpo adiposo bucal, possibilitando seu uso clínico.

A utilização da gordura de Bichat para recobrimento do defeito ósseo e fechamento da comunicação bucosinusal foi selecionada, por ser um método cirúrgico estável, relativamente simples e com altas taxas de sucesso. Sua utilização também foi assegurada pelos autores $^{1,2,3,4,6,10}$, por apresentar um plano tecidual ricamente vascularizado, proporcionando rápida epitelização, em torno de 2 - 6 semanas, menor morbidade ao sítio doador e reduzidas taxas de complicações. Como resultado do caso operado teve-se ótima cicatrização e nenhuma complicação.

De acordo com Egyed $^{9}$, a utilização da gordura de Bichat, para a correção da comunicação bucossinusal, se faz necessária, em casos onde a comunicação enquadre-se na categoria $\mathrm{B}$, ou seja, apresente diâmetro máximo entre 1 a $4 \mathrm{~cm}$. A categoria $A$ e $C$, classificadas como comunicações de até $1 \mathrm{~cm}$ de diâmetro e maiores que $4 \mathrm{~cm}$, respectivamente. Sendo assegurada, também, por Martín-Granizo et al. $^{2}$ e reforçada por este relato, onde a gordura foi utilizada para 0 fechamento de uma comunicação de aproximadamente $1 \mathrm{~cm}$.

O procedimento cirúrgico cominou com o acesso a gordura de Bichat, seu avanço e reposicionamento, recobrindo o defeito ósseo, passivamente. Buscando-se uma melhor estabilidade do tecido avançado, foi realizada uma perfuração óssea, com posterior sutura com monocryl 4-0 (J\&J Medical Devices), mantendo a gordura em posição oclusiva no defeito.

Emes et al. ${ }^{1}$ e Egyed ${ }^{9}$ utilizaram duplo recobrimento da comunicação bucossinusal em seus casos. Inicialmente, o retalho da gordura de Bichat foi reposicionado sobre o defeito ósseo e, posteriormente, foi recoberto com retalho do palato ${ }^{1}$ e da mucosa bucal ${ }^{8}$, obtendo resultados satisfatórios. No presente caso, também se optou pelo fechamento em dupla camada, o que proporcionou um tecido oclusivo mais espesso, bem como acelerou o processo de cicatrização pela não exposição da gordura a cavidade oral, auxiliando 0 processo de metaplasia da gordura.

No caso relatado, optou-se pelo fechamento da comunicação bucossinusal através da gordura de Bichat, por se tratar de uma comunicação de tamanho médio ${ }^{9}$, pelo rico suprimento sanguíneo, facilidade de remoção e por apresentar bons resultados na literatura ${ }^{1,2,3,4,9}$.

Diferente de Martín-Granizo et al. ${ }^{2}$, que ao utilizar a gordura de Bichat com estabilização por sutura em mucosa, obteve epitelização parcial em 1 de seus 29 casos, o presente relato, obteve resultados satisfatório. O paciente foi acompanhado durante 60 dias, apresentando cicatrização em normalidade, sem queixas álgicas ou saída de líquido pela comunicação.

CONSIDERAÇÕES FINAIS

Dessa forma, é evidente os benefícios que a gordura de Bichat apresenta para correção de defeitos ósseos e fechamento de comunicações bucossinusais, devendo ser corretamente indicada, quanto ao diâmetro, localização e a causa do defeito. O conhecimento anatômico e cirúrgico, por parte do cirurgião, é essencial para evitar maiores complicações, por se tratar de uma região correlacionada a estruturas nobres.

\section{REFERÊNCIAS}

1. Emes $Y$, Aga U, Cesur A, Soluk-Tekkesin $M$, Aybar B, Alatli C. Primary closure of oroantral 
communication using pedicled buccal fat pad following maxillary cyst enucleation. J Craniofac Surg. 2018;29(2):e131-33.

2. Martín-Granizo R, Naval L, Costas A, Goizueta C, Rodriguez F, Monje F, Muñoz M, Diaz F. Use of buccal fat pad to repair intraoral defects: review of 30 cases. $\mathrm{Br} \mathrm{J}$ Oral Maxillofac Surg. 1997;35(2):81-4.

3. Mannelli G, Arcuri F, Comini LV, Valente D, Spinelli G. Buccal fat pad: report of 24 cases and literature review of 1,635 cases of oral defect reconstruction. ORL J Otorhinolaryngol Relat Spec. 2019;81(1):24-35.

4. Denes SA, Tieghi R, Elia G. The buccal fat pad for closure of oroantral communication. J Craniofac Surg. 2016;27(3):e327-30.

5. Procacci $P$, Alfonsi $F$, Tonelli $P$, Selvaggi $F$, Menchini Fabris GB, Borgia V, et al. Surgical treatment of oroantral communications. J Craniofac Surg. 2016;27(5):1190-96.

6. Goudar SA, Kumar R, Rathod D, Padmanabhan, Wagdargi SS. Buccal fat pad: a review. Ann Essences Dent. 2014;6(1):37.

7. Heister L. Compendium Anatomicurn. Norimbearge, 1732. Cited in Tharanon W., Stella JP., Epker BN. Applied Surgical Anatomy of the Buccal Fat Pad. Oral Maxillofac Surg Clin North Am 1990;2:377.

8. Bichat FMX. Anatomie Generale: Appliquee a la Physiologie et la Medecine. Paris, 1801. Cited in Stuzin JM, Wagstrom L, Kawamoto HK, Baker TJ, Wolfe A. The Anatomy and Clinical Applications of the Buccal Fat Pad. Plast Reconstr Surg 1990;85:29.

9. Egyedi P. Utilization of the buccal fat pad for closure of oro-antral and/or oro-nasal communications. J Maxillofac Surg. 1977;5(4):241-44.

10. Vuillemin T, Raveh J, Ramon Y. Reconstruction of the maxilla with bone grafts supported by the buccal fat pad. J Oral Maxillofac Surg. 1988;46(2):100-6.

11. Tideman $H$, Bosanquet $A$, Scott $J$. Use of the buccal fat pad as a pedicled graft. J Oral Maxillofac Surg. 1986;44(6):435-40.

\section{CONFLITO DE INTERESSES}

Os autores declaram não haver conflitos de interesse

\section{AUTOR PARA CORRESPONDÊNCIA}

\section{Emerson Filipe de Carvalho Nogueira}

Centro Universitário Brasileiro, UNIBRA

Rua Padre Inglês, 257 - Boa Vista

50050-230 Recife - PE, Brasil

Telefone: +55 (81) 3036-0001

E-mail: emerson_filipe@hotmail.com

Submetido em 12/08/2020

Aceito em 23/10/2020 